\title{
Repetition of color Doppler ultrasonography: is it necessary?
}

\author{
E Akkuş, B Alıcı, H Özkara, S Ataus, M Baǧişgil and H Hattat \\ Department of Urology, Sexual Dysfunctions Center, Cerrahpaşa School of Medicine, 34303 Cerrahpaşa, \\ İstanbul University, Istanbul, Turkey
}

\begin{abstract}
The aim of the study was to determine whether any difference would occur in peak systolic velocity (PSV) and end diastolic velocity (EDV) measurements of color Doppler ultrasonography (CDU) between proximal and distal segments of the cavernous arteries obtained at different times. Twenty-six cases have undergone CDU three times in weekly intervals. PSV and EDV measurements of cavernous arteries were obtained both proximally and distally.

The results have shown that statistically there was no difference between each measurement obtained on either sides at three different tests. (ANOVA PSV $F_{(2-50)}=0.63$, EDV $F_{(2-50)}=0.81$ ). Four of the 26 cases had different PSV results and six cases had different EDV results in three CDU tests. There was a statistical difference in measurements between proximal and distal segments of the cavernous arteries. ( $t$-test $t=2.53, P=0.015$ )

In conclusion, repetition of CDU would not be necessary in majority of the cases. However investigators should always be aware and cautious about falsely abnormal results of CDU. Proximal segments of the cavernous arteries should be the site to obtain true CDU measurements.
\end{abstract}

Keywords: color Doppler ultrasonography; peak systolic velocity; end diastolic velocity

\section{Introduction}

Vascular problems are the most common causes of organic impotence. The initial radiological investigation method used to assess men with suspected vasculogenic impotence is color Doppler ultrasonography of the penile vascular system. Penile anatomy and arterial blood flow through cavernous arteries was first demonstrated with duplex ultrasonography by Lue et al. in $1985 .{ }^{1}$ Initial difficulties in identification of the vessels and maintaining the optimal Doppler angle have been overcome by Quam et $a l^{2}$ with the addition of color flow. Since then many investigators have presented their studies with color doppler ultrasonography in the diagnosis of impotence. Although arteriography is considered the gold standard in the diagnosis of arteriogenic impotence, due to its invasiveness it is not been used in routine practice. Arteriography is indicated in selected cases who are candidates for revascularization procedures or may be suggested in cases with possible intrinsic disease in penile arteries not detected by CDU and in cases with decreased distal penile arterial flow due to other factors such as low cardiac output or increased blood viscosity. As a noninvasive imaging modality

Correspondence: Dr E Akkuş.

Received 4 December 1996; revised 28 March 1997; accepted 21 May 1997 for the functional evaluation of penile flow, CDU is widely being used even as an office based diagnostic tool. Even though the criteria and numerical values of interpreting the measurements of color Doppler ultrasonography (CDU) have varied among centers, CDU is currently considered superior to arteriography as a means of evaluating the arteriogenic component of vascular impotence while its role in the assessment of corporeal veno-occlusive mechanism has not yet been unanimously accepted. ${ }^{3-6}$

There is also evidence that in some cases there may have been falsely abnormal CDU results due to the suppression of response to intracavernous pharmacological agents because of anxiety and increased sympathetic stimulation. ${ }^{7,8}$

The aim of our study was to check the validity of CDU by repeating the investigation three times and to search whether repetition of CDU is necessary or not to obtain a true result of this diagnostic test.

\section{Material and methods}

A total of 26 patients presenting with erectile dysfunctional complaints to our sexual dysfunctions center were included in our study group. Assessment of clinical history, physical examination, hormone levels and intracavernous pharmacotest of papaverine + phentolamine were performed. Those 26 patients who were nonrespon- 
ders to intracavernous pharmacotest with sufficient erection who had normal physical examination and hormone levels were selected to be investigated by CDU. CDUs were performed independently by the same investigator (EA) without the knowledge of the intracavernous pharmacotest and clinical history of the patient which was previously recorded by another investigator.

CDU was performed using Acuson 128X/P10 ultrasound with an imaging frequency of $7.5 \mathrm{Mhz}$ to assess the vascular flow through the cavernous arteries before and after intracavernous injection of $0.2 \mathrm{ml}$ of a tri-mix solution containing $1.8 \mu \mathrm{g}$ PGE-1, $0.2 \mathrm{mg}$ phentolamine and $4.8 \mathrm{mg}$ papaverine. ${ }^{9} \mathrm{CDU}$ was begun in flaccid state of the penis. Then after the intracavernous injection, the patient was asked to stimulate his penis manually in privacy in a comfortable room to induce maximum arterial flow and erection. Complete ultrasonography to evaluate the anatomy and echogenic structure of the penis was performed dorsally, ventrally and laterally. Cavernous arteries were identified along the entire course bilaterally from proximal to distal shaft of the penis and measurements were obtained. Angle of the Doppler cursor was always $50^{\circ}$ for accurate angle corrected velocity calculation and being comparable among different patients. Post injection measurements were obtained starting with the $5 \mathrm{~min}$ of intracavernous injection and manual stimulation and continued to be recorded in 10, 20 and $30 \mathrm{~min}$. Peak systolic velocity (PSV), end diastolic velocity (EDV) measurements were determined and recorded. PSV equal to or greater than $30 \mathrm{~cm} / \mathrm{s}$ and EDV less than $5 \mathrm{~cm} / \mathrm{s}$ were considered normal to exclude vascular diseases. The investigation was repeated two more times within seven days of intervals. Erectile response after injection and manual stimulation was evaluated according to $\mathrm{E}_{1}-\mathrm{E}_{3} \cdot \mathrm{E}_{1}$ : Minimal or no response, with minimal tumescence; $\mathrm{E}_{2}$ : Insufficient erection, tumescence without rigidity; $E_{3}$ : Full erection with full rigidity. At the end of the examinations the patients were informed and reminded about the possibility of prolonged erections due to the intracavernous injections.

Statistical analysis were assessed by Anova test and $t$-test.

\section{Results}

Fifteen of the patients had normal PSV ( $>30 \mathrm{~cm} / \mathrm{s}$ ). Seven of the cases had pathological PSV values on all of the three CDU investigations. Two patients had normal PSV in two tests and pathological PSV on the third. Similarly two patients had abnormal values in two of the tests and a normal PSV value on the third. (Table 1)

Table 1 Results of PSV in 3 CDU

\begin{tabular}{lc}
\hline PSV & Cases \\
\hline All normal in 3 CDU & 15 \\
All abnormal in CDU & 7 \\
Normal in 2, abnormal in 1 CDU & 2 \\
Normal in 1, abnormal in 2 CDU & 2 \\
Total & 26 \\
\hline
\end{tabular}

Table 2 Results of EDV in 3 CDU

\begin{tabular}{lc}
\hline$E D V$ & Cases \\
\hline All normal in 3 CDU & 11 \\
All abnormal in CDU & 9 \\
Normal in 2, abnormal in 1 CDU & 3 \\
Normal in 1, abnormal in 2 CDU & 3 \\
Total & 26 \\
\hline
\end{tabular}

Regarding the EDV values, 11 of the 26 cases had EDV values of $<5 \mathrm{~cm} / \mathrm{s}$, and 9 had $>5 \mathrm{~cm} / \mathrm{s}$ values of EDV in all three CDU examinations. In three cases EDV values were normal in two and pathological in one of the examinations. Similarly three cases had abnormal EDV results in two and normal EDV values in one of the examinations. (Table 2)

PSV values were determined on both (right and left) cavernous arteries, proximally and distally. Twenty-six cases with right and left cavernous arteries times three examinations results with 156 PSV values. In 123 out of 156 (78.8\%), proximal and distal segments of the cavernous arteries revealed different PSV values. In 33 out of 156 (21\%) proximal and distal segments of the arteries revealed the same PSV measurements. In 80 PSV values both proximal and distal segments of the arteries revealed normal results. Both proximal and distal segment revealed abnormal results in $42 \mathrm{PSV}$ values. In 34 out of $156(21.8 \%)$ proximal PSV values were normal while the distal values had $<30 \mathrm{~cm} / \mathrm{s}$ PSV.

When right and left cavernous arteries were compared with CDU results, only in one case PSV revealed normal values on one side and abnormal on the other, meaning unilateral arterial insufficiency. This result was confirmed on all three examinations of that particular case. In the remaining 25 cases both right and left cavernous arteries revealed parallel PSV measurements.

These results have shown that there was no statistical difference between each measurement obtained at three different CDU examinations. ANOVA test for PSV, $F_{(2-50)}=0.63$ and for EDV, $\mathrm{F}_{(2-50)}=0.81$

There was a statistical difference in measurements between proximal and distal segments of the cavernous arteries. ( $t$-test for independent samples $t=2.53$ and $P=0.015$ )

The quality of erections during CDU after intracavernous injections are shown on Table 3. 
Table 3 Measurements of PSV in proximal and distal segment of the cavernous arteries

\begin{tabular}{lcr}
\hline & Number of cases & $\%$ \\
\hline Proximal and distal PSV the same & 33 & 21.2 \\
Proximal and distal PSV different & 123 & 78.8 \\
Total & $156^{\mathrm{a}}$ & 100.0 \\
\hline
\end{tabular}

${ }^{\mathrm{a}} 26$ cases $\times 2$ (right and left arteries) $\times 3$ tests $=156$.

Table 4 Results of PSV in proximal and distal segments of the cavernous arteries

\begin{tabular}{lcc}
\hline$P S V$ & Number of tests & $\%$ \\
\hline Proximal normal distal normal & 80 & 51.3 \\
Proximal abnormal distal abnormal & 42 & 26.9 \\
Proximal normal distal abnormal & 34 & 21.8 \\
Total & 156 & 100.0 \\
\hline
\end{tabular}

Table 5 Quality of erections during 3 CDU

\begin{tabular}{lc}
\hline Erections & $\begin{array}{c}\text { Number of } \\
\text { cases }\end{array}$ \\
\hline $\mathrm{E}_{1}$ in all 3 tests & 1 \\
$\mathrm{E}_{2}$ in all 3 tests & 7 \\
$\mathrm{E}_{3}$ in all 3 tests & 5 \\
$\mathrm{E}_{1}$ in $2, \mathrm{E}_{2}$ in 1 test & 2 \\
$\mathrm{E}_{1}$ in $1, \mathrm{E}_{2}$ in 2 tests & 3 \\
$\mathrm{E}_{1}$ in $1, \mathrm{E}_{3}$ in 2 tests & 1 \\
$\mathrm{E}_{1}$ in $1, \mathrm{E}_{2}$ in $1, \mathrm{E}_{3}$ in 1 test & 1 \\
$\mathrm{E}_{2}$ in $2, \mathrm{E}_{3}$ in 1 test & 3 \\
$\mathrm{E}_{2}$ in $1, \mathrm{E}_{3}$ in 2 tests & 3 \\
Total & 26 \\
\hline
\end{tabular}

During CDU test one case had three $\mathrm{E}_{1}$, seven cases had three $E_{2}$ and 5 cases had three $E_{3}$ constantly after intracavernous injections and manual stimulation. Two of the cases had two $E_{1}-1 E_{2}$, four cases had one $E_{1}-2 E_{2}$, and one case had $E_{2}-2 E_{3}$, three cases had two $E_{2}-1 E_{3}$, and three cases had one $\mathrm{E}_{2}-2 \mathrm{E}_{3}$ during the CDU test.

\section{Discussion}

Vascular abnormalities are the most common cause of impotence and their precise diagnosis is very important when choosing the adequate therapy for erectile dysfunction. Introduction of duplex ultrasonography with color flow doppler analysis after intracavernous injection of vasoactive drugs has been reported to be a minimally invasive and accurate method and considered to be the gold standard technique to evaluate penile hemodynamics. ${ }^{1,10}$ CDU of the cavernous arteries provide functional, quantifiable assessment of penile arterial flow during a pharmacological erection. However
Repetition of color Doppler ultrasonography

E Akkus et al

investigators are aware that there are cases which result in a false abnormal CDU studies. It is presumed that anxiety and increased sympathetic stimulation result in a falsely abnormal response to pharmacological stimulation and subsequent CDU measurements. ${ }^{7,8}$

Our results with the repetition of CDU three times in weekly intervals have shown that in 22 out of 26 cases PSV measurements did not change the diagnostic values. The values of PSV were different only in four cases leading to doubts in the diagnosis with CDU. Similarly in 6 out of 26 cases EDV measurements were contradictory when CDU was performed three times. Statistical interpretation of the above results revealed that there was no statistical difference between each measurements obtained in three examinations. Nevertheless the investigators should still be cautious evaluating abnormal CDU results.

Even though uncertainties still exist over the optimal combination and doses to be used in intracavernous injections ${ }^{9}$ in our experience $0.2 \mathrm{ml}$ intracavernous injection of tri-mix combination with adequate sexual stimulation provides sufficient smooth muscle relaxation and arterial dilatation to obtain valid CDU values. There should be no doubt that manual stimulation of the penis after intracavernous injection is imperative for optimal erectile response and accurate CDU measurements. ${ }^{11,12}$

The role of EDV to evaluate the veno-occlusive mechanism by CDU is still debatable. ${ }^{2-5,13,14}$ Currently the gold standart to diagnose veno-occlusive dysfunction (VOD) is obviously pharmacocavernosometry/graphy. However EDV of $<5 \mathrm{~cm} / \mathrm{s}$ will avoid investigators to perform unnecessary pharmacocavernosometry/graphy. ${ }^{8}$ There is no doubt that pharmacocavernosometry/graphy is necessary if EDV $>5 \mathrm{~cm} / \mathrm{c}$ on CDU which still exist even after 20-30 min after the intracavernous injection in cases we would plan penile venous surgery. In 20 out of 26 cases EDV values in three consecutive CDU examinations were parallel which was also statistically meaningfull. Nevertheless in our opinion we should still be cautious to interpret the EDV measurements as suspect values in the diagnosis of VOD regarding 6 out of 26 cases with contradictory results in three consecutive CDU tests.

Our study has shown that proximal and distal segments of the cavernous arteries have different values of vascular flow. Seventy-nine percent of the PSV values in our series revealed different values proximally vs distally, which was also statistically significant. Nineteen percent of the PSV values have shown that variations in measurements of proximal and distal segments of the cavernous arteries may lead to contradictory diagnosis. Therefore the appropriate site to detect the PSV is the proximal site of the cavernous artery rather than the distal segment. 
It has been reported that the association of multiple intracorporeal injections and stimulations increase the diagnostic potential of CDU. ${ }^{13,15}$ Four of our 26 cases $(15.4 \%)$ PSV values were contradictory leading to misdiagnosis. This inaccuracy may be overcome by repeating intracorporeal injections followed by genital and audiovisual sexual stimulations. Also it has been previously reported that psychogenic impotence cases may have falsely abnormal response to pharmacological stimulation and CDU. ${ }^{8}$ Thus in cases with suspected psychogenic impotence, CDU values must be interpreted cautiously perhaps as in our four cases. It seems that repetition of CDU after pyschological consultation and therapy in such cases may avoid false abnormal results of CDU and thus the diagnosis of impotence. Our four cases of different PSV values and six cases with different EDV measurements in three different CDU tests have shown that in such suspected cases with psychogenic inhibition, CDU test may be repeated. Because CDU is a noninvasive diagnostic tool, repetition of the test should not be avoided in such cases. Otherwise nocturnal penile tumescence (NPT) test may be performed to provide a better diagnostic information. ${ }^{7}$

Our study has once again shown that there is always a possibility of unilateral vascular disease in the cavernous arteries. One of our 26 cases $(3.8 \%)$ was such an example of this possibility. Therefore measurements of CDU should always be performed bilaterally on both cavernous arteries.

The quality of erections postinjectively during the test have also shown some variations. Thirteen of the 26 cases $(50 \%)$ in our series experienced different quality of erections even in the same kind and dosage of the pharmacological agent, manual stimulation and the same circumstances where CDUs were performed. Particularly one of the cases who had no response to intracavernous injection in the first test have experienced two good sufficient erections in the second and third tests. Another one of our cases have experienced an improvement in each of the consecutive tests by no response in the first CDU, insufficient erection in the second and good erection in the third test. The interpretation of this variations in the quality of erections during CDU tests is possibly due to the anxiety in the initial test and relaxation in the following tests by the recognition that CDU is not an invasive method. Also the influence of intracavernosally injected drugs might possibly be increased in the consecutive tests and revealed better smooth muscle relaxation and arterial dilatation leading to better quality of erections.

In terms of deciding the sensitivity and specificity of our study, the results need to be compared to arteriography. However due to its invasiveness and limited indications, we did not perform arteriography in our series. This is probably the caveat of our study.

\section{Conclusions}

Repetition of CDU would not be necessary in majority of the cases. However investigators must always be aware and cautious about the possibility of falsely abnormal results with CDU particularly in psychogenically effected cases, and repeat CDU when they think it should. Repeating the injections and sexual stimulations during CDU may also increase the diagnostic value of CDU. Likewise poor quality of erections during CDU may not be the same in repetition of the test and therefore should not rule out a possible self-injection therapy alternative. Proximal segment of the cavernous arteries should be the site to obtain the hemodynamic measurements to prevent the possible misdiagnosis when distal segments were measured. Finally a thorough understanding of erectile physiology and anatomy is necessary to properly perform and interpret CDU.

To our knowledge no report has been published concerning the validity of CDU by repeating the test. Therefore we do not have the opportunity to compare our results with other investigators. Further similar repeated studies in the future will possibly be able to compare the results of CDU tests.

\section{References}

1 Lue TF, Hricak H, Marich KW, Tanagho EA. Vasculogenic impotence evaluated by high-resolution ultrasonography and pulsed Doppler spectrum analysis. Radiology 1985; 155: 777.

2 Quam JP et al. Duplex and color Doppler sonograhic evaluation of vasculogenic impotence. AJR 1989; 153: 11411147.

3 Kropman RF et al. The role of increased end diastolic velocity during penile duplex sonography in relation to pathological venous leakage in erectile dysfunction. J Urol 1992; 148: 314317.

4 Bassiouny HS, Levine LA. Penile duplex sonograhy in the diagnosis of venogenic impotence. J Vasc Surg 1991; 13: 7583.

5 Nisen HO, Saarinen O, Ruutu ML, Edgren J. Duplex Doppler scanning with prostaglandin E1 in the diagnosis of cavernous leakage. Acta Rad 1993; 34: 335-338.

6 Merck LA, De Bruyne RMG, Goes E, Derde MP, Keuppens F. The value of dynamic color duplex scanning in the diagnosis of venogenic impotence. J Urol 1992; 148: 318-320.

7 Allen RP, Engel RME, Smolev JK, Brendler CB. Comparison of duplex sonography and nocturnal penile tumescence in the evaluation of impotence. J Urol 1994; 151: 1525-1529.

8 Mellinger BC, Vaughan ED Jr. Penile blood flow changes in the flaccid and erect state in the potent young men measured by duplex scanning. J Urol 1990; 144: 894-896.

9 Goldstein I et al. Rescuing the failed papaverine/phentolamine erection: A proposed synergistic action of papaverine, phentolamine and prostaglandine E1. Int J Impot Res 1990; 2 (suppl 2): 277.

10 Broderick GA, Arger P. Duplex Doppler sonography: Noninvasive assessment of penile anatomy and function. Sem Roentgenol 1993; 28: 43-46.

11 Benson GS. Vascular evaluation: Is it useful in 1992? In: Lue TF, ed. World Book of Impotence, Smith Gordon and Co: London, 1992, pp 85-90. 
12 Donatucci CF, Lue TF. The combined intracavernous injection and stimulation test: Diagnostic accuracy. J Urol 1992; 148: 61-63.

13 Montorsi $\mathrm{F}$ et al. The effect of intracorporeal injection plus genital and audiovisual sexual stimulation versus second injection on penile color Doppler sonography parameters. J Urol 1996; 155: 536-540.
Repetition of color Doppler ultrasonography

E Akkus et al

14 Vickers MA, Benson CB, Richie JP. High resolution ultrasonography and pulsed wave Doppler for detection of corporavenous incompetence in erectile dysfunction. J Urol 1990; 143: 1125-1127.

15 Lue TF. Personal communication.

\section{Editorial comment}

\section{Repetition of color Doppler ultrasonography: is it necessary?- by E Akkuş et al}

Penile Duplex Ultrasonography (PPDU) is used to assess the arterial- and veno-occlusive response in men with erectile dysfunction. To date, in a goaldirected approach its application is limited to research protocols and as a screening test in patients in whom a reconstructible vascular disease is suspected. Its value as a screening tool in a general population of men with erectile dysfunction is considered limited due to the lack of therapeutic consequences. Because, the veno-occlusive mechanism is highly susceptible to psychological factors and to the type and dosage of the vasoactive agent, the reproducibility of the veno-occlusive response is poor. In contrast, the reproducibility of the arterial response is relatively good because it is less dependent on psychological factors and the dosage of the vasoactive agent.

Of course, other methodological factors such as the sampling location and interval after pharmacological stimulation are critical. It has been well documented that there is a mean reduction of flow velocity

\section{Response to editorial comment}

I totally agree with Dr Meuleman's comment emphasizing the caveats of Color Doppler Ultrasonography (CDU) in the diagnosis of impotence. Our aim in the study was to raise the question of whether CDU results are always consistent or not, due to the psychologically inhibiting factors and/or introcavernous injections during CDU. between the crural and distal subcoronal cavernous artery of about $20 \%$, whereas in patients with peripheral arterial occlusive disease, velocity may be reduced $50 \%$. With respect to location, the consensus in the literature is that velocity tracings should be obtained in the most proximal part of the cavernous arteries. With respect to timing, the consensus is that arterial response is to be determined in the phase of erection with highest flow rates, namely in the first minutes following pharmacological stimulation. In the literature controversies exist on the significance of unilateral abnormalities and reference values. For example, the cut-off value for peak flow velocity vary between $20-35 \mathrm{~cm} / \mathrm{s}$ and may be age-dependent. Probably, the truth is somewhere in the middle. In a good scientific tradition the article of Akkus and coworkers raises a small corner of the veil and simultaneously poses new questions.

EJH Meuleman WL Diemont

Our opinion is to repeat CDU whenever a suspect might arise due to the abovementioned factors affecting the results of the investigation and subsequently the diagnosis.

Thank you Dr Diemont and Dr Meuleman for your kind comments.

E Akkuş, MD 